\title{
Multipole Radiations from Large Gold Nanospheres Excited by Evanescent Wave
}

\author{
Jingdong Chen ${ }^{1,2}{ }^{\oplus}$, Jin Xiang ${ }^{1}{ }^{\oplus}$, Shuai Jiang ${ }^{1}$, Qiaofeng Dai ${ }^{1}$, Shaolong Tie $^{3}$ and \\ Sheng $\operatorname{Lan}^{1, *(1)}$ \\ 1 Guangdong Provincial Key Laboratory of Nanophotonic Functional Materials and Devices, School of \\ Information and Optoelectronic Science and Engineering, South China Normal University, \\ Guangzhou 510006, China; jdchen@163.com (J.C.); xiangcos@163.com (J.X.); jiangs1224@163.com (S.J.); \\ daiqf@scnu.edu.cn (Q.D.) \\ 2 College of Physics and Information Engineering, Minnan Normal University, Zhangzhou 363000, China \\ 3 School of Chemistry and Environment, South China Normal University, Guangzhou 510006, China; \\ tiesl@scnu.edu.cn \\ * Correspondence: slan@scnu.edu.cn
}

Received: 11 January 2019; Accepted: 26 January 2019; Published: 31 January 2019

\begin{abstract}
We proposed the use of the evanescent wave generated in a total internal reflection configuration to excite large gold nanospheres and investigated the radiations of the high-order plasmon modes supported in gold nanospheres. It was revealed that the evanescent wave excitation is equivalent to the excitation by using both the incident and reflected light, offering us the opportunity to control the orientation of the electric field used to excite nanoparticles. In addition, it was found that the scattering light intensity is greatly enhanced and the background noise is considerably suppressed, making it possible to detect the radiations from high-order plasmon modes. Moreover, the influence of the mirror images on the scattering induced by a metal substrate is eliminated as compared with the surface plasmon polariton excitation. By exciting a gold nanosphere with $s$-polarized light and detecting the scattering light with a $p$-polarized analyzer, we were able to reveal the radiation from the electric quadrupole mode of the gold nanosphere in both the spatial and the frequency domains. Our findings are important for characterizing the radiations from the high-order modes of large nanoparticles and useful for designing nanoscale photonic devices.
\end{abstract}

Keywords: gold nanosphere; electric dipole; electric quadrupole; electric octupole; plasmon mode; scattering; evanescent wave; radiation pattern

\section{Introduction}

Surface plasmons supported in noble metallic nanostructures have formed a highly attractive branch of nanoscale optical physics in the last two decades. Due to the excitation of collective coherent quantized oscillations of conduction band electrons in the metal nanostructures, both the absorption and scattering cross-sections are significantly enlarged compared with their physical dimensions, resulting in a strong light-matter interaction, subwavelength volume confinement, and optical field enhancement [1,2]. Owing to the superior properties of surface plasmons, various potential applications have been inspired including surface-enhanced Raman spectroscopies [3-7], ultrasensitive biosensing [8-10], fluorescence enhancement [11,12], photothermal therapy [13-15], ultrahigh density data storage [16,17], nonlinear optics conversion [18-21], and quantum optics [22], etc.

Of the various nanostructures, spherical nanoparticles are one of the most fundamental and important ones that possess the highest geometrical symmetry and could act as the simplest isotropic antenna. More interestingly, one can construct more complicated and sophisticated nanostructures 
(e.g., dimers, oligomers, and particle-on-film [23-34]) which function as photonic devices by using spherical nanoparticles as building blocks. Based on Mie theory [35], the far-field scattering of a plasmonic spherical particle can be analytically decomposed into the contributions of multipoles. With increasing diameter, the electric dipole (ED) of the nanoparticle will be redshifted to the near-infrared spectral range while the high-order plasmon modes, such as electric quadrupole (EQ) and electric octupole (EOC), will emerge in the visible light spectrum. For instance, the high-order plasmon modes of spherical gold mesoparticles are experimentally observed by using reflectance spectroscopy [36]. Owing to the broad linewidth of these plasmon modes, the coherent interaction between them can be employed to realize highly directional scattering, delicate beam shaping, and effective wavefront control $[34,37,38]$. For example, it was shown that highly directional plasmonic nanoantennas can be realized by exploiting the constructive interference between the ED and EQ of a nano-ring when the generalized Kerker condition is satisfied [38,39]. This intriguing behavior indicates the crucial role of the high-order plasmon modes in engineering the far-field radiation in the nanoscale plasmonic structures. Owing to the inherent Ohmic loss of metals, the linewidth of the ED resonance is much broader than those of the high-order plasmon modes, implying the importance of the high-order plasmon modes in practical applications where an enhancement in the electric field is required, such as surface-enhanced hyper-Raman scattering [6,7]. Therefore, the investigation of the high-order plasmon modes induced in metallic nanospheres, especially the EQ mode, is quite important for designing photonic devices capable of manipulating light radiation and emission.

So far, dark-field microscopy is the most popular way to characterize the far-field scattering of nanoparticles. Although it is a simple and effective method to measure the forward/backward scattering of nanoparticles, there are still some limitations. For example, an objective with a small numerical aperture (NA) is generally employed in a dark-field microscope to collect the scattering light in order to exclude the illumination light. Thus, only a small fraction of the scattering light is detected, leading to a weak scattering intensity or a low signal-to-noise ratio. Moreover, it is not convenient to control the polarization of the illumination light and thus the orientation of the electric field used to excite the nanoparticle. In our previous work [40], we used the surface plasmon polaritons generated on the surface of a thin gold film to excite the gold nanospheres (GNSs) placed on the thin film and investigated the radiation behaviors of various plasmon modes excited in large GNSs. In this case, the scattering intensity of a GNS is greatly enhanced due to the strongly localized electric field in the surface plasmon polaritons, offering us the opportunity to investigate the radiations from the high-order plasmon modes, such as the electric quadrupole (EQ) and electric octupole (EOC) modes. In addition, a completely dark background is achieved in the total internal reflection configuration utilized to excite the surface plasmon polaritons, resulting in radiation patterns with high contrasts. Unfortunately, there are still three drawbacks in this method. Since the surface plasmon polaritons can only be created by using p-polarized, the polarization control of the illumination light is strongly limited. Moreover, the existence of the thin gold film will introduce mirror images for all plasmon modes and the scattering of a GNS is finally determined by the coherent interaction of the plasmon modes and their mirror images, making the spatial and frequency analysis of the scattering behavior more complicated. Finally, two gap plasmon modes, which are oriented horizontally and vertically, are introduced in between the GNS and the thin gold film and contribute to the total scattering of the GNS. Apart from conventional dark-field microscopy, scanning nearfield optical microscopy [41,42], photoelectron emission microscopy [43-45], and electron microscopy [46-48] have also been employed for optical field imaging at the nanoscale and even spectrum recording.

Very recently, we proposed the use of the evanescent wave generated in the total internal reflection configuration to excite silicon nanoparticles for a color-tuning display with a high spatial resolution and a good chromaticity [49]. In this strategy, the orientations of the electric and magnetic fields used to excite the Si nanoparticle can be conveniently adjusted by simply varying the polarization angle of the incident light. The excitation of a nanoparticle by using such an evanescent wave is equivalent to the excitation of the nanoparticle by using both the incident and the reflected light. The scattering intensity 
of the nanoparticle is significantly enhanced while the background noise is considerably suppressed. Compared to the surface plasmon polaritons, the influence of the mirror images is eliminated and the polarization control of the excitation light becomes possible and convenient.

In this article, we employed the evanescent wave generated in the total internal reflection configuration to excite the GNSs placed on a silica substrate. The high-order plasmon modes excited in the GNS were investigated by exciting the GNSs with s- and p-polarized light and detecting the scattering light of the GNSs with a cross-polarized analyzer. We revealed the radiation from the quadrupole of a large GNS in both frequency and spatial domains by using s-polarized evanescent wave excitation in combination with a cross-polarized analyzer in the collection channel. We also indicate that highly directional radiation may be realized by exploiting the coherent interaction between the ED and EQ modes of the GNS.

\section{Experimental and Numerical Methods}

\subsection{Sample Preparation}

We employed femtosecond (fs) laser ablation to fabricate GNSs with diameters ranging from 80-560 nm. In experiments, as schematically shown in Figure 1a, 800-nm fs laser light delivered by a fs amplifier (Legend Elite, Coherent, Santa Clara, CA, USA) with a pulse duration of $90 \mathrm{fs}$ and a repetition rate of $1 \mathrm{kHz}$ was focused on the surface of a 50-nm-thick Au film by using a lens with 22-mm focal length. The ejected GNSs with different diameters were randomly distributed on a silica substrate covered on the Au film. The morphologies and sizes of the GNSs were examined by using a scanning electron microscope (SEM) (Ultra55, Zeiss, Stutt-gart, Germany).

\subsection{Optical Characterization}

The scattering properties of GNSs excited either by using the white light in a conventional dark-field microscope or by using the evanescent wave generated in a total internal reflection configuration were characterized by using an inverted microscope (Axio Observer A1, Zeiss, Stutt-gart, Germany) equipped with a spectrometer (SR-500i-B1, Andor, Oxford, England) and a full-color coupled charge device (CCD) (DS-Ri2, Nikon, Tokyo, Japan). In the conventional dark-field microscopy, the white light was incident on the Au film at an angle of $\sim 58^{\circ}$ with respect to the normal of the surface. In comparison, $s$ - and $p$-polarized white light was launched into a prism at an incidence angle of $\sim 45^{\circ}$ to excite the evanescent wave. The silica substrate was attached on the bottom surface of the prism with silicon oil for refractive index matching. The scattering light of GNSs was collected with a $100 \times$ objective (NA = 0.7-1.3, Plan-NEOFWAR, Zeiss, Stutt-gart, Germany) in the forward direction and analyzed by inserting a polarization analyzer in the collecting channel.

\subsection{Numerical Modeling}

The scattering spectra of GNSs were either analytically calculated based on Mie theory or numerically simulated by using the finite-difference time-domain (FDTD) technique. The multipolar contributions to the total scattering of the GNSs are calculated analytically and numerically. The permittivity of Au was fitted from experimental data [50]. For the simulation of the scattering spectra of GNSs excited by the white light in the conventional dark-field microscopy, we used a total-field/scattered-field source to simulate the scattering efficiencies of GNSs excited by a plane wave incident normally on the silica substrate. Differently, a plane wave with an incidence angle of $\sim 45^{\circ}$ was employed to simulate the scattering spectra of GNSs excited by the evanescent wave, as schematically shown in Figure 1b. In the numerical simulations, evanescent waves were automatically created on the surface of the silica substrate when the condition of total internal reflection was satisfied. A cubic monitor with a volume of 8000 (length) $\times 8000$ (width) $\times 1600$ (height) $\mathrm{nm}^{3}$ enclosing the GNSs was used to record the electromagnetic field distributions. A standard two-step method in which the total scattering field and background field in the absence of GNSs were extracted separately was employed 
to calculate the net scattering fields of GNSs excited by the evanescent waves. Based on the net scattering fields, one can obtain the far-field scattering properties of GNSs under the excitation of the evanescent wave. In order to compare the simulation results with the experimental observations, we detected only the scattering in the forward direction by using a monitor with an area of 1000 (length) $\times 1000$ (width) $\mathrm{nm}^{2}$ in the calculation of the scattering cross-section which offers a collection angle quite similar to that provided by the objective with NA $=0.7$ and index-match oil ( 1.4). Differently, the net electromagnetic field distributions from all six monitors were extracted to obtain the far-field radiation patterns. Based on the calculated scattering fields and the Poynting vectors, in addition to their components, the scattering cross-sections and far-field radiation patterns of GNSs excited by the evanescent with or without a polarization analyzer could be obtained. The mesh size used in the GNS was set to be smaller than 1/60 of its radius. A perfectly matched layer boundary condition was employed to terminate the finite simulation region.

\section{Results and Discussion}

\subsection{Dark-Field Microscopy}

The scattering properties of large GNSs prepared by using fs laser ablation were firstly examined by conventional dark-field microscopy, as schematically shown in Figure 1c. The scattering spectra measured for GNSs with diameters ranging from 140 to $350 \mathrm{~nm}$ are presented in Figure $1 \mathrm{~d}$. The simulation results based on the FDTD technique in which the silica substrate is taken into account are also provided for comparison. The SEM images of the GNSs and the radiation patterns of the GNSs recorded by using a CCD are shown as insets.

Based on Mie theory, the EQ and EOC modes emerge in the visible light spectrum when the diameter of the GNS becomes larger than 220 and $380 \mathrm{~nm}$, respectively. Since the refractive index of silica is small ( 1.45), its influence on the multipole resonances of Au nanospheres is quite small. In Figure 1e, we show the scattering spectrum analytically calculated for the GNS with $d=350 \mathrm{~nm}$ based on Mie theory. It has been decomposed into the contributions of ED, EQ, and EOC modes. It is noticed that the ED and EQ modes of the GNS appear at 980 and $585 \mathrm{~nm}$, respectively. Owing to the large linewidths of the ED and EQ resonances, the spectral overlapping of the ED and EQ modes occurs in a wide wavelength range of 500-820 nm. It implies that the scattering of the GNS in this wavelength range is governed by the coherent interaction of the ED and EQ modes. As a consequence, the forward scattering at the EQ resonance becomes stronger than that at the ED resonance, see Figure 1d. In Figure 1f, we present the 2D and 3D radiation patterns of the GNS calculated at two wavelengths of 980 and $630 \mathrm{~nm}$. At $980 \mathrm{~nm}$ where the ED resonance is located, we observed a radiation pattern of a typical ED mode oriented along the $x$ direction; although, it is slightly distorted due to the existence of a small amount of the EQ mode. In this case, the intensities of the forward and backward scattering are almost the same. In sharp contrast, it is found that the forward scattering is significantly enhanced while the backward scattering is greatly suppressed at $630 \mathrm{~nm}$ where the amplitudes of the ED and EQ resonances are equal. The highly directional radiation achieved in the forward direction originates from the constructive interference between the ED and EQ modes. The simulated radiation pattern at $\sim 630 \mathrm{~nm}$ appears to be quite similar to that obtained by the constructive interference between a dipole and a quadrupole, verifying the physical mechanism for the enhanced forward scattering.

In Figure 1d, the scattering spectra measured for GNSs are in good agreement with those predicted based on numerical simulations except for the wavelengths larger than $800 \mathrm{~nm}$ where the quantum efficiency of the detector drops rapidly. By increasing the diameter of the GNS, one can see a redshift of the scattering peak and a broadening of the scattering spectrum. Since the redshift of the ED resonance is larger than that of the EQ resonance, the coherent interaction between them will become weaker in large GNSs. For the smallest GNS with $d=140 \mathrm{~nm}$, the scattering spectrum is governed by the ED resonance and the scattering light appears as green-yellow. For the GNSs with $d>220 \mathrm{~nm}$, the ED resonance is redshifted to long wavelengths and the scattering spectrum is dominated by the 
EQ resonance appearing in the wavelength range of 550-650 $\mathrm{nm}$. Since the contribution of the ED resonance to the scattering light is still large, the color of the scattering light appears as white. In all cases, the radiation pattern of the GNS appears to be the typical one of a horizontally oriented ED mode (i.e., a bright spot) although it contains the contribution from the EQ mode. It means that the scattering light from the EQ mode cannot be resolved by using conventional dark-field microscopy.

(a)

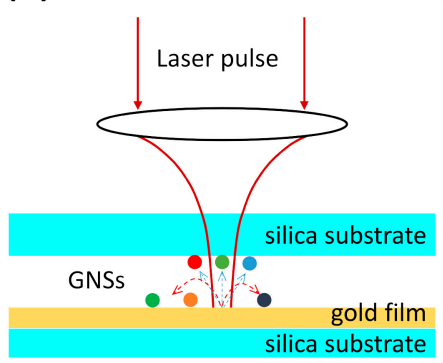

(d)

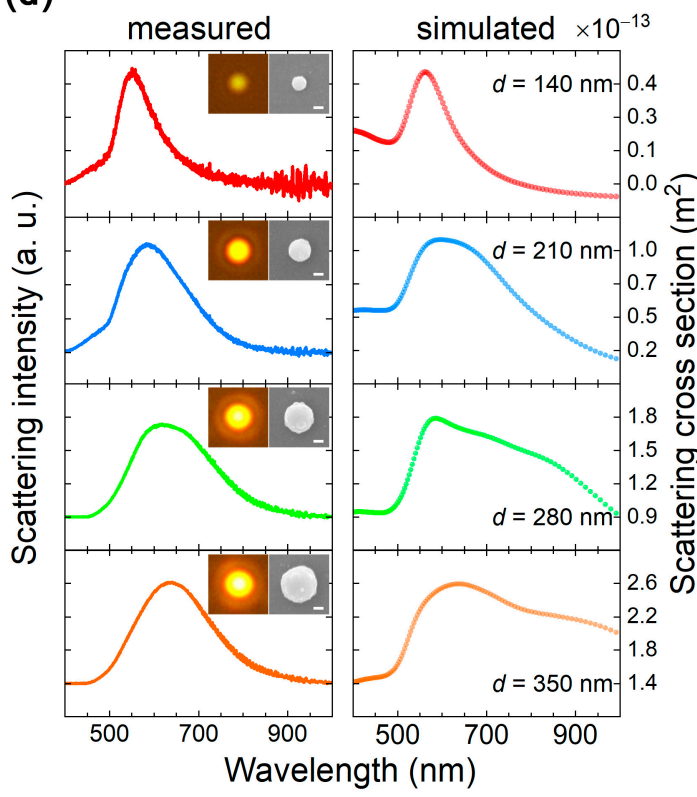

(b)

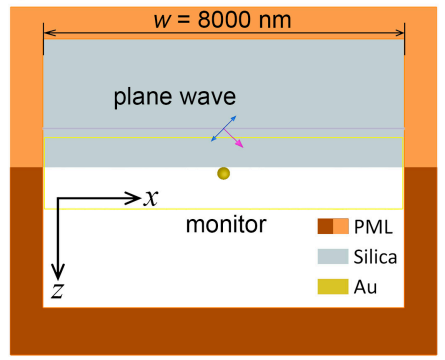

(c)

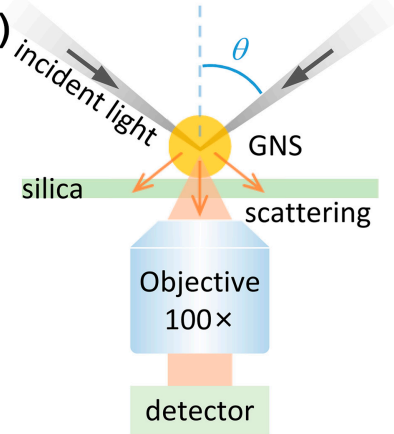

(e)

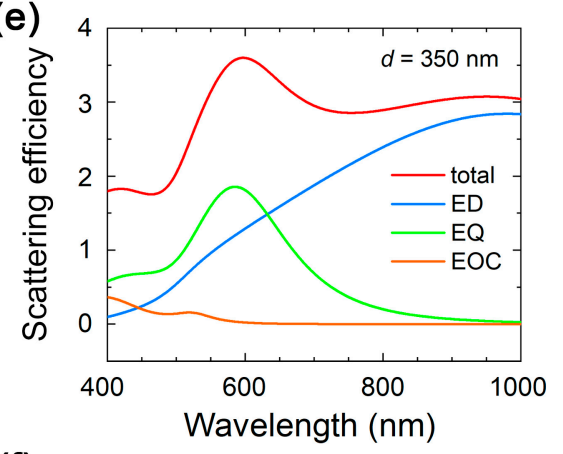

(f)

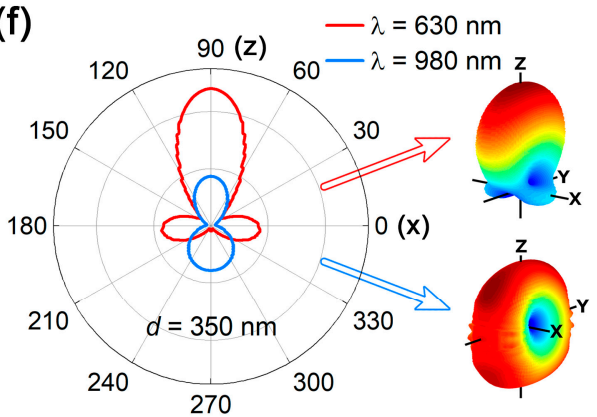

Figure 1. (a) Schematic showing the fabrication of gold nanospheres (GNSs) with different diameters on a silica substrate by using fs laser ablation. (b) Configuration used to simulate the far-field radiation from a GNS excited by an evanescent wave in the finite-difference time-domain (FDTD) simulations. (c) Schematic showing the illumination of a GNS placed on a silica substrate with white light at an incidence angle of $\theta \sim 58^{\circ}$, and the detection of the forward scattering light in a dark-field microscope. (d) Forward scattering spectra measured and simulated for GNSs with different diameters placed on a silica substrate. The SEM images of the corresponding GNSs and the coupled charge device (CCD) images of the scattering light are shown in the insets. The length of the scale bar is $100 \mathrm{~nm}$ in all cases. (e) The total scattering spectrum calculated for a GNS with $d=350 \mathrm{~nm}$ in free space based on Mie theory. The scattering has been decomposed into the contributions of electric dipole (ED), electric quadrupole (EQ), and electric octupole (EOC). (f) Two- and three-dimensional radiation patterns calculated for the GNS with $d=350 \mathrm{~nm}$ in free space at two wavelengths of 630 and $980 \mathrm{~nm}$.

\subsection{Evanescent Wave Excitation}

In order to gain a deep insight into the radiation of the EQ mode of a large GNSs, we employed the evanescent wave generated in a total internal reflection configuration to excite the GNS and 
detected the forward scattering of the GNS with the help of a polarization analyzer, as schematically shown in Figure 2a. The advantages of evanescent wave excitation over the conventional dark-field microscopy are threefold. First, one can choose to excite the GNS with either $p$ - or s-polarized light, making it possible to manipulate the orientation of the electric field used to excite the GNS. Second, the evanescent wave excitation is basically equivalent to the excitation of GNS with both the incident and reflected light with a fixed phase difference. The in-phase overlap of the electric fields of the two-excitation light at the critical angle for total internal reflection leads to a vertically oriented electric field for $p$-polarized light and a horizontally oriented electric field for s-polarized light, as shown in Figure $2 b, c$, respectively. In both cases, the scattering light is enhanced while the background noise is suppressed, improving significantly the signal-to-noise ratio in the measurements. Finally, the excitation of the GNS with polarized light offers the possibility to completely eliminate the radiation of the ED mode by inserting a cross-polarized analyzer into the collection channel [49,51,52], making it possible to resolve the quadrupole scattering in both the frequency and spatial domains.

(a)

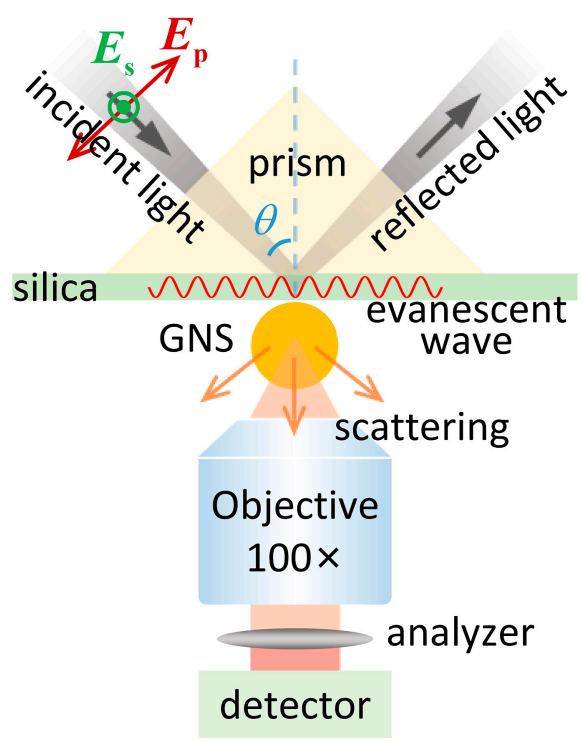

(b)
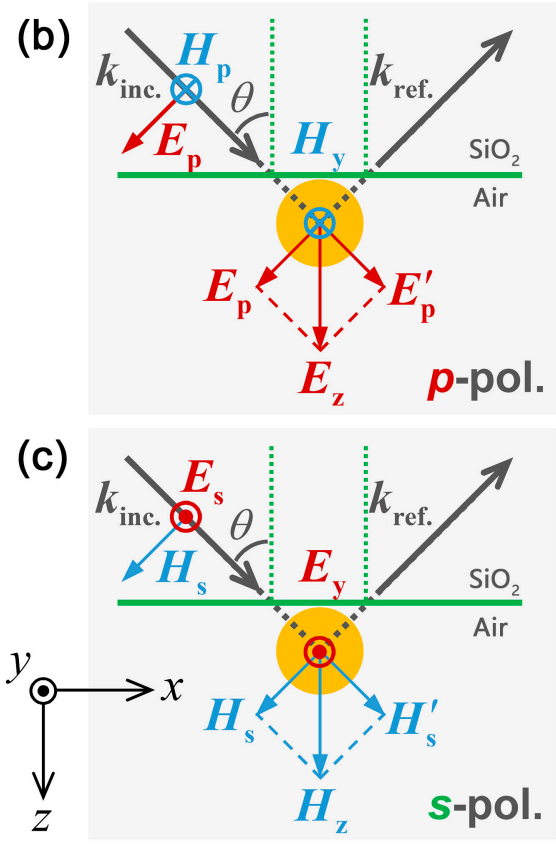

Figure 2. (a) Schematic showing the excitation of a GNS with the evanescent wave generated in a total internal reflection configuration and the detection of the quadrupole radiation of the GNS by filtering out the dipole radiation with a cross-polarized analyzer. The incidence angle is chosen to be $\theta \sim 45^{\circ}$. (b) Schematic showing the excitation of a GNS by using the evanescent wave generated with $p$-polarized light. (c) Schematic showing the excitation of a GNS by using the evanescent wave generated with s-polarized light. In (b) and (c), the combined electric and magnetic fields of the incident and reflected light which determine the orientations of the ED and magnetic dipole (MD) induced in the GNS by $p$ - and s-polarized light are illustrated.

In the experiments, the incidence angle was chosen to be the critical angle for total internal reflection $\left(\sim 45^{\circ}\right)$ so that the reflected light is in phase with the incident one (i.e., the phase difference is approximately zero). In this case, the electric field of the evanescent wave induced by the s-polarized light has only the horizontal component $\left(E_{\mathrm{y}}\right)$ while that induced by the $p$-polarized light has only the vertical component $\left(E_{\mathrm{z}}\right)$. In this way, we can study the scattering properties of a large GNS excited by a horizontally or vertically aligned electric field.

In Figure 3, we show the scattering spectra measured and simulated for a GNS with $d \sim 280 \mathrm{~nm}$ which is excited by using $p$ - and s-polarized light. When the GNS is excited by using $p$-polarized light, the peak of the scattering spectrum appears at $\sim 620 \mathrm{~nm}$. In comparison, the scattering peak of the GNS is redshifted to $\sim 690 \mathrm{~nm}$ with a small shoulder at $\sim 570 \mathrm{~nm}$ when s-polarized is used. It is also 
noticed that the simulated spectrum under the excitation of s-polarized light, shown in Figure 3b, appears to be similar to the one obtained in the conventional dark-field microscopy, see Figure $1 \mathrm{~d}$. This is because the excitation of the GNS achieved by using the evanescent induced by s-polarized light is actually the excitation of the GNS with a horizontally aligned electric field, which is exactly the same as the excitation of the GNS by using a normally incident plane wave. If we compare the scattering patterns recorded by using a CCD in the two cases, however, it is found that the contrast of the CCD image obtained in the evanescent wave excitation is much better than that obtained in the dark-field microscopy. The significantly improved contrast of the CCD image arises from the considerably suppressed background in the generation of the evanescent wave where the total internal reflection configuration is employed.
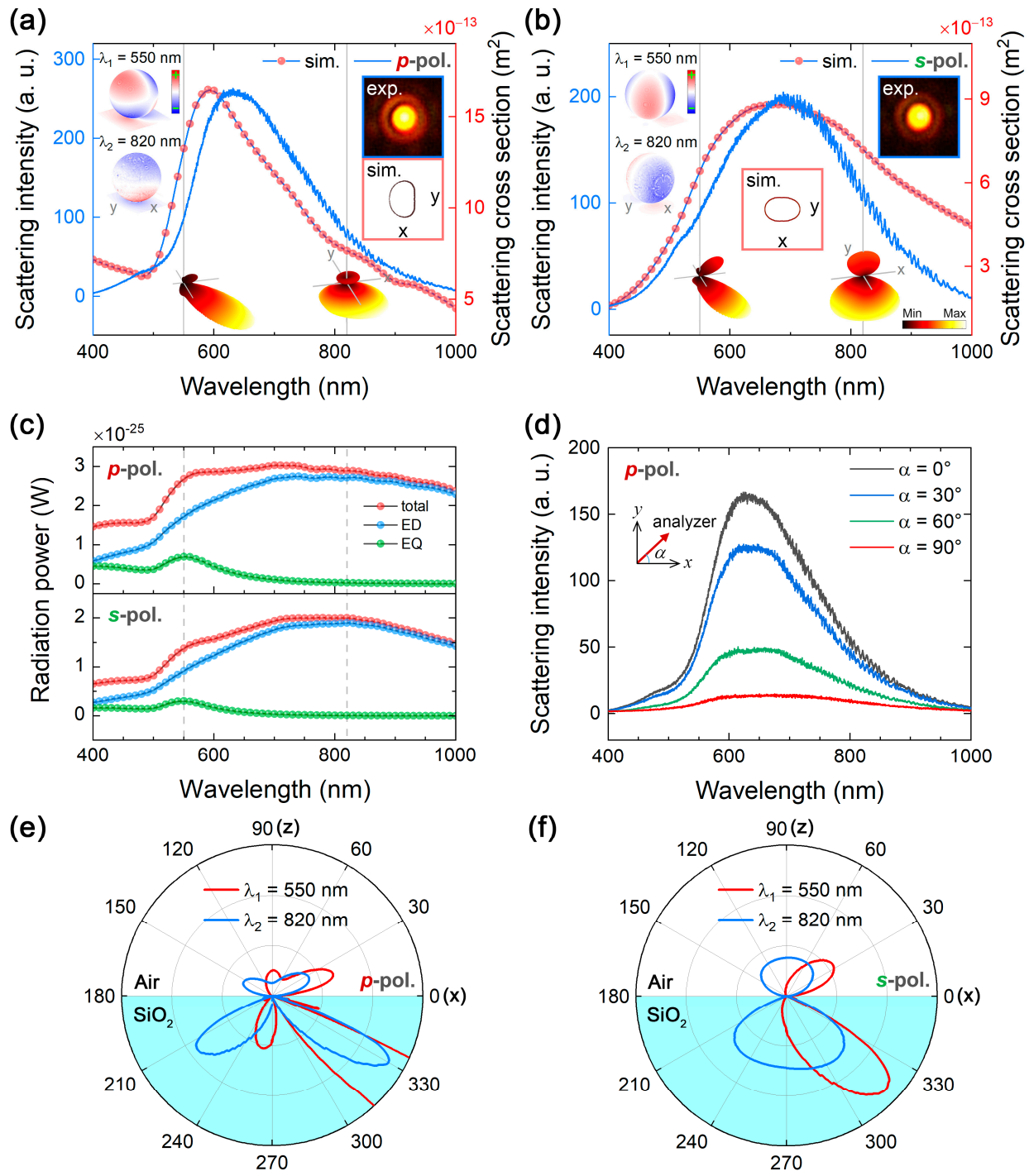

Figure 3. Scattering spectra measured and simulated for a GNS with $d \sim 280 \mathrm{~nm}$ excited by (a) $p$ and (b) s-polarized light. Insets show the calculated three-dimensional far-field radiation patterns at 550 and $820 \mathrm{~nm}$. The surface charge distributions at the two wavelengths are also provided as insets. (c) Multipole expansion of the total scattering of a GNS with $d=280 \mathrm{~nm}$ excited by using $p$ - and s-polarized light. (d) Scattering spectra of the GNS with $d \sim 280 \mathrm{~nm}$ excited by $p$-polarized light and measured by setting the polarization analyzer at different angles $(\alpha)$ of $0^{\circ}, 30^{\circ}, 60^{\circ}$, and $90^{\circ}$. The two-dimensional radiation patterns (on the xoz plane) calculated for the GNS excited by $p$ - and s-polarized light are shown in (e) and (f), respectively. 


\subsection{Multipole Expansion of the Scattering Spectrum}

Since the refractive index of silica is close to that of a vacuum, the existence of the silica substrate has a negligible influence on the plasmon modes excited in a GNS. Since the phase difference between the incident and reflected light is zero at the critical angle of the total internal reflection, the excitation of the GNS by using an evanescent wave is equivalent to the simultaneous excitation of the nanoparticle with two plane waves. Therefore, we employed the multipole expansion method to approximately determine the electric resonances in a GNS excited by an evanescent wave in order to understand the physical origins of the scattering peaks appearing in the scattering spectra of the GNS shown in Figure $3 a, b$. In a Cartesian coordinate, the radiated moments of electric dipole (ED), magnetic dipole (MD), toroidal dipole (TD), electric quadrupole (EQ), and magnetic quadrupole (MQ) can be derived from the induced volume current density $\mathbf{j}$ in the GNS as follows in the SI units [53-56]:

$$
\begin{gathered}
\mathbf{E D}=\frac{1}{i \omega} \int \mathbf{j} d^{3} r \\
\mathbf{M D}=\frac{1}{2 c} \int(\mathbf{r} \times \mathbf{j}) d^{3} r \\
\mathbf{T D}=\frac{1}{10 c} \int\left[(\mathbf{r} \cdot \mathbf{j}) \mathbf{r}-2 r^{2} \mathbf{j}\right] d^{3} r \\
E Q_{\alpha \beta}=\frac{1}{i \omega} \int\left[r_{\alpha} j_{\beta}+r_{\beta} j_{\alpha}-\frac{2}{3}(\mathbf{r} \cdot \mathbf{j})\right] d^{3} r \\
M Q_{\alpha \beta}=\frac{1}{3 c} \int\left[(\mathbf{r} \times \mathbf{j})_{\alpha} r_{\beta}+(\mathbf{r} \times \mathbf{j})_{\beta} r_{\alpha}\right] d^{3} r
\end{gathered}
$$

Here, $c$ is the speed of light in vacuum and $\alpha, \beta=x, y, z$. The total radiating power can then be expressed as:

$$
\begin{aligned}
& I=\frac{2 \omega^{4}}{3 c^{3}}|\mathbf{E D}|^{2}+\frac{2 \omega^{4}}{3 c^{3}}|\mathbf{M D}|^{2}+\frac{4 \omega^{5}}{3 c^{4}}(\mathbf{E D} \cdot \mathbf{T D}) \\
& +\frac{2 \omega^{6}}{3 c^{5}}|T|^{2}+\frac{\omega^{6}}{5 c^{5}} \sum\left|E Q_{\alpha \beta}\right|^{2}+\frac{\omega^{6}}{40 c^{5}} \sum\left|M Q_{\alpha \beta}\right|^{2}
\end{aligned}
$$

We show the decomposed scattering spectra of a GNS with $d=280 \mathrm{~nm}$ excited by using $\mathrm{p}$-and s-polarized light in Figure 3c. For clarity, only the ED and EQ resonances are presented because the contributions of other plasmon modes are negligible. It can be seen that the total scattering of the GNS is dominated by the ED mode with a broad linewidth and a peak at $\sim 820 \mathrm{~nm}$. In comparison, the EQ mode possesses a narrower linewidth and a weak intensity. It is located at $\sim 550 \mathrm{~nm}$, which corresponds to the small shoulder in the scattering spectrum. As compared with the simulated spectra shown in Figure $3 a, b$, it is noticed that the ED resonance is stronger in the near-infrared spectral range because the scattering intensities in all directions are taken into account in the multipole expansion method. In contrast, only the forward scattering is considered in the simulated spectra shown in Figure 3a,b. In this case, highly directional scattering is observed at the EQ resonance when the generalized Kerker condition [39] is satisfied.

\subsection{Revealing High-Order Plasmon Modes}

Based on the multipole decomposition shown in Figure 3c, we were able to determine the peak wavelengths of the EQ and ED modes in the GNS with $d=280 \mathrm{~nm}$, which are located at $\sim 550$ and $\sim 820 \mathrm{~nm}$ (labeled as $\lambda_{1}$ and $\lambda_{2}$, respectively). Surface charge distributions calculated for the GNS at the ED and EQ resonances are presented in the insets of Figure $3 \mathrm{a}, \mathrm{b}$ for $p$ - and s-polarized light excitations. For the $p$-polarized light excitation, a vertically aligned ED mode is identified from the surface charge distribution on the GNS at $820 \mathrm{~nm}$. In addition, a typical EQ mode is revealed from the surface charge distribution at $550 \mathrm{~nm}$. For the s-polarized light excitation, the surface charge distribution at $820 \mathrm{~nm}$ indicates a horizontally aligned ED mode, while that at $550 \mathrm{~nm}$ displays a horizontally oriented EQ mode. The surface charge distributions on the GNS at the ED and EQ resonances agree well with the 
combined electric fields induced by the incident and reflected light shown in Figure 2b,c. It verifies that a horizontally and vertically aligned electric field can be selectively created by using the evanescent wave generated in the total internal reflection configuration. The orientations of the plasmon modes can be experimentally identified by analyzing the polarization of the scattering light. For example, the scattering intensities of the ED and EQ modes of the GNS with $d \sim 280 \mathrm{~nm}$ excited by $p$-polarized light decreased as the angle of the polarization analyzer was increased from $0^{\circ}$ to $90^{\circ}$, as shown in Figure 3d. However, the ED mode located at a longer wavelength was not completely removed because it orients vertically.

In Figure 3e,f, we present the two-dimensional (2D) far-field radiation patterns on the $x o z$ plane calculated at wavelengths of 820 and $550 \mathrm{~nm}$ for $p$ - and s-polarized light. The three-dimensional (3D) radiation patterns are also provided in the insets of Figure 3a,b. In Figure 3a, the radiation pattern at $\lambda_{2}=820 \mathrm{~nm}$ is dominated by a vertically oriented ED. Owing to the existence of the silica substrate, the radiation intensity into the substrate is much stronger than that into air. At $\lambda_{1}=550 \mathrm{~nm}$, the radiation pattern is determined by the coherent interaction between EQ and ED modes. From the 2D pattern on the $x o z$ plane shown in Figure $3 \mathrm{e}$, it can be seen that the scattering light in the $+z$ direction (from $60^{\circ}$ to $120^{\circ}$, corresponding to the NA of the objective) is dominated by the EQ mode. As a result, the calculated and simulated scattering spectra are dominated by the EQ mode rather than the ED mode when the GNS is excited by using $p$-polarized light, as shown in Figure 3a. In contrast, the radiation pattern of the ED mode is dramatically changed when the GNS is excited by using s-polarized light, as shown in the inset of Figure $3 b$. The existence of the silica substrate significantly modifies the radiation of a horizontally oriented ED mode, see the inset of Figure $1 \mathrm{f}$. The radiation pattern is quite similar to the result reported previously [34]. In addition, the radiation of the EQ mode in the $+z$ direction is greatly reduced, as clearly shown in Figure 3f. In this case, only a small fraction of the EQ mode is collected in both the measurement and the simulation. Consequently, the measured scattering spectrum is dominated by the ED mode rather than the EQ mode because the collection angle of the objective is $\sim 60^{\circ}$, as reflected in Figure $3 \mathrm{~b}$.

Since the scattering light comprises the radiations from the ED and EQ modes, it is necessary to eliminate the radiation of the ED mode in order to reveal the radiation properties of the EQ mode in both the spatial and frequency domains. In Figure $4 a, b$, we show the scattering spectra and radiation patterns measured for a GNS with $d \sim 280 \mathrm{~nm}$. In this case, the GNS was excited by $p$-polarized light and the scattering light was detected by inserting a $p$ - or s-polarized analyzer in the collection channel. When the GNS is excited by using $p$-polarized light, only the vertical component of the electric field is left, see Figure $2 b$. In this case, the radiation of the excited ED exhibits a doughnut-shaped ring while that of the EQ mode exhibits a butterfly-like shape. For the vertically oriented ED $\left(\lambda_{2}=820 \mathrm{~nm}\right)$, most of the scattering light is removed when a $p$ - or s-polarized analyzer is used. As a result, one can see two spots along the polarization direction of the analyzer. The 2D pattern on the $x o z$ or yoz plane is shown by the blue curve in Figure $4 \mathrm{~b}$. As compared with the case without the use of a polarization analyzer, see Figure 3a, the scattering intensity is reduced to some extent. For the EQ mode, the use of an s-polarized analyzer is completely different from the use of a $p$-polarized one. When a $p$-polarized analyzer was used, the radiation intensity was reduced but the radiation pattern remained unchanged, as shown by the red curve in the top panel of Figure $4 \mathrm{~b}$. The radiation pattern recorded by the CCD appeared as a bright spot, in good agreement with the simulated one. When an s-polarized analyzer was used, the radiation in the $+z$ direction was completely removed at 550 and $820 \mathrm{~nm}$, as shown in the lower panel of Figure $4 b$. From the 3D radiation pattern of the EQ mode shown in Figure $4 a$, it can be seen that the radiation in the upper part is almost eliminated. From the bottom panel of Figure $4 b$, it is observed that only two lobes are left in the 2D radiation pattern of the EQ mode after the use of the s-polarized analyzer, similar to the ED mode. In this case, the scattering intensity is significantly reduced because most of the scattering light is not collected by the objective. The radiation pattern exhibited two lobes along the $y$-axis. 
(a)

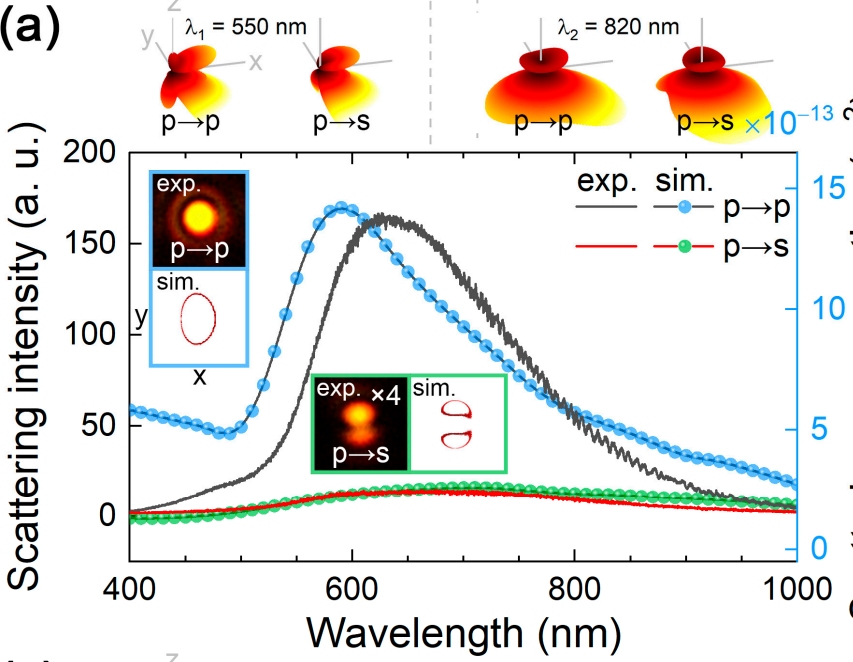

(c)

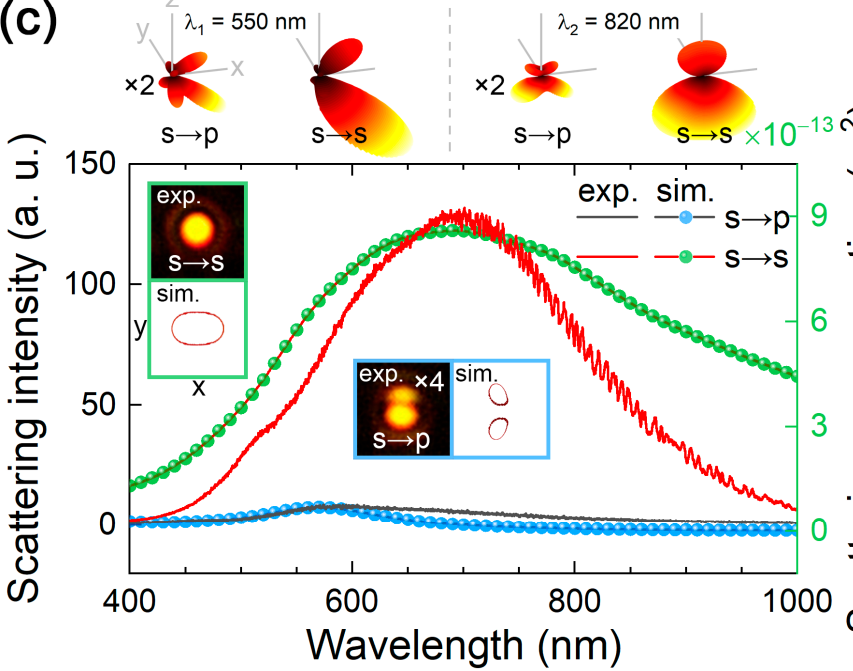

(b)

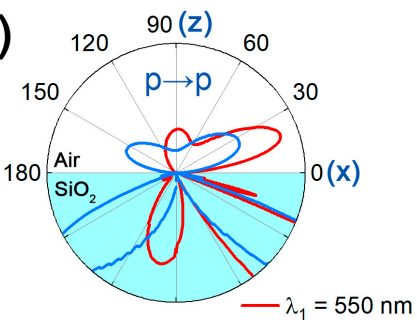

心

朋

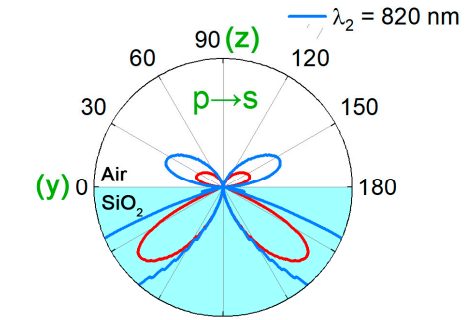

(d)

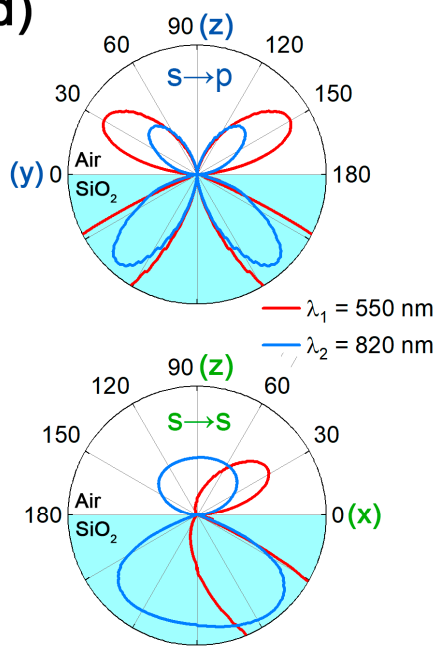

Figure 4. Scattering spectra measured and simulated for a GNS with $d \sim 280 \mathrm{~nm}$, which is excited by using $p$ - and $s$-polarized light and detected by using a $p$ - and s-polarized analyzer. (a) Excited by $p$-polarized light and detected by using a $p$ - and s-polarized analyzer. (b) Two-dimensional radiation patterns on the $x o z$ and yoz planes of the GNS excited by using $p$-polarized light at 550 and $820 \mathrm{~nm}$ and detected by using a $p$ - and s-polarized analyzer. (c) Excited by s-polarized light and detected by using a $p$ - and s-polarized analyzer. (d) Two-dimensional radiation patterns on the $x o z$ and yoz planes of the GNS excited by using s-polarized light 550 and $820 \mathrm{~nm}$ detected by using a $p$-/s-polarized analyzer. The two-dimensional radiation patterns recorded by using a CCD and those on the xoy plane and the three-dimensional radiation calculated by using the FDTD method are shown in the insets of (a) and (c).

The most interesting phenomenon is observed when the GNS was excited by using s-polarized light and the scattering light was measured by using a p-polarized analyzer, as shown in Figure 4c. In this case, the radiation of the excited ED mode, which is horizontally oriented, is completely filtered out by the cross-polarized analyzer inserted into the collection channel. As a result, we observed the scattering spectrum and radiation pattern of a pure EQ mode. The scattering spectrum was dominated by the EQ resonance peak at $~ 550 \mathrm{~nm}$. Accordingly, the 2D patterns (on the yoz plane) at both 550 and $820 \mathrm{~nm}$ exhibit the radiation of a typical EQ mode because of its broad linewidth, implying that the ED mode has been completely removed. When an s-polarized analyzer was used, the scattering spectrum was governed by the strong radiation of the ED mode peaking at $\sim 820 \mathrm{~nm}$, as shown in the bottom panel of Figure $4 \mathrm{~d}$. Consequently, a bright spot was observed in the radiation which is typical for a horizontally oriented ED mode. Therefore, the observation of the radiation of a pure EQ mode can 
only be realized by exciting the GNS with s-polarized light and detecting the scattering light with a p-polarized analyzer.

\section{Conclusions}

In summary, we have fabricated GNSs with diameters ranging from 80 to $560 \mathrm{~nm}$ by using femtosecond laser ablation and proposed the use of evanescent wave excitation to investigate the radiations from the high-order plasmon modes excited in GNSs. The use of evanescent wave excitation makes it possible to easily control the orientation of the electric field used to excite GNSs, significantly enhancing the scattering light intensity and considerably reducing the background noise. In addition, the influence of the mirror images on the radiation behaviors of plasmon modes is also eliminated. More importantly, the radiation from the high-order plasmon modes of a GNS, such as the EQ mode, can be revealed in both the spatial and the frequency domains by exciting the GNS with s-polarized light and detecting the scattering light by using a $p$-polarized analyzer. The evanescent wave excitation proposed in this work is quite useful for characterizing the scattering properties of nanoparticles, especially the high-order plasmon modes. The highly directional radiations resulting from the coherent interaction of the plasmon modes may be exploited to design nanoantennas.

Author Contributions: J.C., J.X. and S.L. conceived the idea; J.C. and J.X. performed the numerical simulation; J.C., J.X., S.J., Q.D. and S.T. fabricated samples and conducted the optical measurements. J.C. and S.L. wrote the manuscript. S.L. supervised the project. All authors consulted their results and have read, critically reviewed, and agreed to the final version of the manuscript.

Funding: Sheng Lan and Shaolong Tie acknowledge the financial supports from the National Nature and Science Foundation of China (Grant Nos. 11674110 and 11874020), the Natural Science Foundation of Guangdong Province, China (Grant No. 2016A030308010), and the Science and Technology Planning Project of Guangdong Province, China (Grant No. 2015B090927006).

Conflicts of Interest: The authors declare no conflict of interest.

\section{References}

1. Barnes, W.L.; Dereux, A.; Ebbesen, T.W. Surface Plasmon Subwavelength Optics. Nature 2003, 424, 824-830. [CrossRef] [PubMed]

2. Halas, N.J.; Lal, S.; Chang, W.-S.; Link, S.; Nordlander, P. Plasmons in Strongly Coupled Metallic Nanostructures. Chem. Rev. 2011, 111, 3913-3961. [CrossRef] [PubMed]

3. Zhang, R.; Zhang, Y.; Dong, Z.C.; Jiang, S.; Zhang, C.; Chen, L.G.; Zhang, L.; Liao, Y.; Aizpurua, J.; Luo, Y.; et al. Chemical Mapping of a Single Molecule by Plasmon-Enhanced Raman Scattering. Nature 2013, 498, 82-86. [CrossRef] [PubMed]

4. Ding, S.Y.; Yi, J.; Li, J.F.; Ren, B.; Wu, D.Y.; Panneerselvam, R.; Tian, Z.Q. Nanostructure-Based Plasmon-Enhanced Raman Spectroscopy for Surface Analysis of Materials. Nat. Rev. Mater. 2016, 1, 16021. [CrossRef]

5. Chen, S.; Zhang, Y.; Shih, T.M.; Yang, W.; Hu, S.; Hu, X.; Li, J.; Ren, B.; Mao, B.; Yang, Z.; et al. Plasmon-Induced Magnetic Resonance Enhanced Raman Spectroscopy. Nano Lett. 2018, 2209-2216. [CrossRef] [PubMed]

6. Itoh, T.; Yoshikawa, H.; Yoshida, K.; Biju, V.; Ishikawa, M. Evaluation of Electromagnetic Enhancement of Surface Enhanced Hyper Raman Scattering Using Plasmonic Properties of Binary Active Sites in Single Ag Nanoaggregates. J. Chem. Phys. 2009, 130. [CrossRef] [PubMed]

7. Kneipp, K.; Kneipp, H.; Itzkan, I.; Dasari, R.R.; Feld, M.S. Surface-Enhanced non-Linear Raman Scattering at the Single-Molecule Level. Chem. Phys. 1999, 247, 155-162. [CrossRef]

8. Larsson, E.M.; Alegret, J.; Kall, M.; Sutherland, D.S. Sensing Characteristics of NIR Localized Surface Plasmon Resonances in Gold Nanorings for Application as Ultrasensitive Biosensors. Nano Lett. 2007, 7, 1256-1263. [CrossRef] [PubMed]

9. Schasfoort, R.B.M.; Abali, F.; Stojanovic, I.; Vidarsson, G.; Terstappen, L. Trends in SPR Cytometry: Advances in Label-Free Detection of Cell Parameters. Biosensors 2018, 8, 102. [CrossRef] 
10. Saha, K.; Agasti, S.S.; Kim, C.; Li, X.; Rotello, V.M. Gold Nanoparticles in Chemical and Biological Sensing. Chem. Rev. 2012, 112, 2739-2779. [CrossRef]

11. Kinkhabwala, A.; Yu, Z.F.; Fan, S.H.; Avlasevich, Y.; Mullen, K.; Moerner, W.E. Large Single-Molecule Fluorescence Enhancements Produced by a Bowtie Nanoantenna. Nat. Photonics 2009, 3, 654-657. [CrossRef]

12. Akselrod, G.M.; Argyropoulos, C.; Hoang, T.B.; Ciraci, C.; Fang, C.; Huang, J.; Smith, D.R.; Mikkelsen, M.H. Probing the Mechanisms of Large Purcell Enhancement in Plasmonic Nanoantennas. Nat. Photonics 2014, 8, 835-840. [CrossRef]

13. Yao, Y.; Zhang, N.; Liu, X.; Dai, Q.; Liu, H.; Wei, Z.; Tie, S.; Li, Y.; Fan, H.; Lan, S. A Novel Fast Photothermal Therapy Using Hot Spots of Gold Nanorods for Malignant Melanoma Cells. Nanomaterials 2018, 8, 880. [CrossRef] [PubMed]

14. Goel, S.; Ferreira, C.A.; Chen, F.; Ellison, P.A.; Siamof, C.M.; Barnhart, T.E.; Cai, W. Activatable Hybrid Nanotheranostics for Tetramodal Imaging and Synergistic Photothermal/Photodynamic Therapy. Adv. Mater. 2018, 30, 1704367. [CrossRef] [PubMed]

15. Huang, X.; El-Sayed, I.H.; Qian, W.; El-Sayed, M.A. Cancer Cell Imaging and Photothermal Therapy in The Near-Infrared Region by Using Gold Nanorods. J. Am. Chem. Soc. 2006, 128, 2115-2120. [CrossRef] [PubMed]

16. Dai, Q.; Ouyang, M.; Yuan, W.; Li, J.; Guo, B.; Lan, S.; Liu, S.; Zhang, Q.; Lu, G.; Tie, S.; et al. Encoding Random Hot Spots of a Volume Gold Nanorod Assembly for Ultralow Energy Memory. Adv. Mater. 2017, 29, 1701918. [CrossRef] [PubMed]

17. Zijlstra, P.; Chon, J.W.; Gu, M. Five-Dimensional Optical Recording Mediated by Surface Plasmons in Gold Nanorods. Nature 2009, 459, 410-413. [CrossRef]

18. Li, J.X.; Xu, Y.; Dai, Q.F.; Lan, S.; Tie, S.L. Manipulating Light-Matter Interaction in a Gold Nanorod Assembly by Plasmonic Coupling. Laser Photonics Rev. 2016, 10, 826-834. [CrossRef]

19. Shen, S.; Meng, L.; Zhang, Y.; Han, J.; Ma, Z.; Hu, S.; He, Y.; Li, J.; Ren, B.; Shih, T.M.; et al. Plasmon-Enhanced Second-Harmonic Generation Nanorulers with Ultrahigh Sensitivities. Nano Lett. 2015, 15, 6716-6721. [CrossRef]

20. Wang, H.; Huff, T.B.; Zweifel, D.A.; He, W.; Low, P.S.; Wei, A.; Cheng, J.X. In Vitro and in Vivo Two-Photon Luminescence Imaging of Single Gold Nanorods. Proc. Natl. Acad. Sci. USA 2005, 102, 15752-15756. [CrossRef]

21. Kim, S.; Jin, J.; Kim, Y.J.; Park, I.Y.; Kim, Y.; Kim, S.W. High-Harmonic Generation by Resonant Plasmon Field Enhancement. Nature 2008, 453, 757-760. [CrossRef] [PubMed]

22. Zhu, W.; Esteban, R.; Borisov, A.G.; Baumberg, J.J.; Nordlander, P.; Lezec, H.J.; Aizpurua, J.; Crozier, K.B. Quantum Mechanical Effects in Plasmonic Structures with Subnanometre Gaps. Nat. Commun. 2016, 7, 11495. [CrossRef] [PubMed]

23. Prodan, E.; Radloff, C.; Halas, N.J.; Nordlander, P. A Hybridization Model for the Plasmon Response of Complex Nanostructures. Science 2003, 302, 419-422. [CrossRef] [PubMed]

24. And, P.N.; Oubre, C.; Prodan, E.; And, K.L.; Stockman, M.I. Plasmon Hybridization in Nanoparticle Dimers. Nano Lett. 2004, 4, 899-903. [CrossRef]

25. Nordlander, P.; Prodan, E. Plasmon Hybridization in Nanoparticles Near Metallic Surfaces. Nano Lett. 2004, 4, 2209-2213. [CrossRef]

26. Brown, L.V.; Sobhani, H.; Lassiter, J.B.; Nordlander, P.; Halas, N.J. Heterodimers: Plasmonic Properties of Mismatched Nanoparticle Pairs. ACS Nano 2010, 4, 819-832. [CrossRef] [PubMed]

27. Li, G.-C.; Zhang, Y.-L.; Lei, D.Y. Hybrid Plasmonic Gap Modes in Metal Film-Coupled Dimers and Their Physical Origins Revealed by Polarization Resolved Dark Field Spectroscopy. Nanoscale 2016, 8, 7119-7126. [CrossRef] [PubMed]

28. Li, G.C.; Zhang, Y.L.; Jiang, J.; Luo, Y.; Lei, D.Y. Metal-Substrate-Mediated Plasmon Hybridization in a Nanoparticle Dimer for Photoluminescence Line-Width Shrinking and Intensity Enhancement. ACS Nano 2017, 3067-3080. [CrossRef] [PubMed]

29. Verellen, N.; Sonnefraud, Y.; Sobhani, H.; Hao, F.; Moshchalkov, V.V.; Van Dorpe, P.; Nordlander, P.; Maier, S.A. Fano Resonances in Individual Coherent Plasmonic Nanocavities. Nano Lett. 2009, 9, 1663-1667. [CrossRef]

30. Mirin, N.A.; Bao, K.; Nordlander, P. Fano Resonances in Plasmonic Nanoparticle Aggregates. J. Phys. Chem. A 2009, 113, 4028-4034. [CrossRef] 
31. Fan, J.A.; Bao, K.; Wu, C.; Bao, J.; Bardhan, R.; Halas, N.J.; Manoharan, V.N.; Shvets, G.; Nordlander, P.; Capasso, F. Fano-Like Interference in Self-Assembled Plasmonic Quadrumer Clusters. Nano Lett. 2010, 10, 4680-4685. [CrossRef] [PubMed]

32. Fan, J.A.; Wu, C.H.; Bao, K.; Bao, J.M.; Bardhan, R.; Halas, N.J.; Manoharan, V.N.; Nordlander, P.; Shvets, G.; Capasso, F. Self-Assembled Plasmonic Nanoparticle Clusters. Science 2010, 328, 1135-1138. [CrossRef] [PubMed]

33. Lei, D.Y.; Fernandez-Dominguez, A.I.; Sonnefraud, Y.; Appavoo, K.; Haglund, R.F.; Pendry, J.B.; Maier, S.A. Revealing Plasmonic Gap Modes in Particle-on-Film Systems Using Dark-Field Spectroscopy. ACS Nano 2012, 6, 1380-1386. [CrossRef] [PubMed]

34. Zhang, Q.; Li, G.C.; Lo, T.W.; Lei, D.Y. Polarization-Resolved Optical Response of Plasmonic Particle-on-Film Nanocavities. J. Opt. 2018, 20, 024010. [CrossRef]

35. Mie, G. Beiträge zur Optik trüber Medien, speziell kolloidaler Metallösungen. Ann. Phys. 1908, 330, 377-445. [CrossRef]

36. Evlyukhin, A.B.; Kuznetsov, A.I.; Novikov, S.M.; Beermann, J.; Reinhardt, C.; Kiyan, R.; Bozhevolnyi, S.I.; Chichkov, B.N. Optical Properties of Spherical Gold Mesoparticles. Appl. Phys. B 2012, 106, 841-848. [CrossRef]

37. Coenen, T.; Bernal Arango, F.; Femius Koenderink, A.; Polman, A. Directional Emission from a Single Plasmonic Scatterer. Nat. Commun. 2014, 5, 3250. [CrossRef] [PubMed]

38. Alaee, R.; Filter, R.; Lehr, D.; Lederer, F.; Rockstuhl, C. A Generalized Kerker Condition for Highly Directive Nanoantennas. Opt. Lett. 2015, 40, 2645-2648. [CrossRef]

39. Kerker, M.; Wang, D.S.; Giles, C.L. Electromagnetic Scattering by Magnetic Spheres. J. Opt. Soc. Am. 1983, 73, 765-767. [CrossRef]

40. Chen, J.D.; Xiang, J.; Jiang, S.; Dai, Q.F.; Tie, S.L.; Lan, S. Radiation of the High-Order Plasmonic Modes of Large Gold Nanospheres Excited by Surface Plasmon Polaritons. Nanoscale 2018, 10, 9153-9163. [CrossRef]

41. Bouillard, J.S.; Vilain, S.; Dickson, W.; Zayats, A.V. Hyperspectral Imaging with Scanning Near-Field Optical Microscopy: Applications in Plasmonics. Opt. Express 2010, 18, 16513-16519. [CrossRef] [PubMed]

42. Liu, N.; Li, Z.; Xu, H. Polarization-Dependent Study on Propagating Surface Plasmons in Silver Nanowires Launched by a Near-Field Scanning Optical Fiber Tip. Small 2012, 8, 2641-2646. [CrossRef] [PubMed]

43. Cinchetti, M.; Gloskovskii, A.; Nepjiko, S.A.; Schonhense, G.; Rochholz, H.; Kreiter, M. Photoemission Electron Microscopy as a Tool for the Investigation of Optical Near fields. Phys. Rev. Lett. 2005, 95, 047601. [CrossRef]

44. Grubisic, A.; Ringe, E.; Cobley, C.M.; Xia, Y.; Marks, L.D.; Van Duyne, R.P.; Nesbitt, D.J. Plasmonic Near-Electric Field Enhancement Effects in Ultrafast Photoelectron Emission: Correlated Spatial and Laser Polarization Microscopy Studies of Individual Ag Nanocubes. Nano Lett. 2012, 12, 4823-4829. [CrossRef] [PubMed]

45. Word, R.C.; Fitzgerald, J.; Koenenkamp, R. Photoelectron Emission Control with Polarized Light in Plasmonic Metal Random Structures. Appl. Phys. Lett. 2011, 99, 041106. [CrossRef]

46. Vogelgesang, R.; Dmitriev, A. Real-Space Imaging of Nanoplasmonic Resonances. Analyst 2010, 135, 1175-1181. [CrossRef]

47. Sun, Q.; Yu, H.; Ueno, K.; Kubo, A.; Matsuo, Y.; Misawa, H. Dissecting the Few-Femtosecond Dephasing Time of Dipole and Quadrupole Modes in Gold Nanoparticles Using Polarized Photoemission Electron Microscopy. ACS Nano 2016, 10, 3835-3842. [CrossRef]

48. Thollar, Z.; Wadell, C.; Matsukata, T.; Yamamoto, N.; Sannomiya, T. Three-Dimensional Multipole Rotation in Spherical Silver Nanoparticles Observed by Cathodoluminescence. ACS Photonics 2017, 5, 2555-2560. [CrossRef]

49. Xiang, J.; Li, J.T.; Zhou, Z.P.; Jiang, S.; Chen, J.D.; Dai, Q.F.; Tie, S.L.; Lan, S.; Wang, X.H. Manipulating the Orientations of the Electric and Magnetic Dipoles Induced in Silicon Nanoparticles for Multicolor Display. Laser Photonics Rev. 2018, 12, 1800032. [CrossRef]

50. Johnson, P.B.; Christy, R.W. Optical Constants of the Noble Metals. Phys. Rev. B 1972, 6, 4370-4379. [CrossRef]

51. Permyakov, D.; Sinev, I.; Markovich, D.; Ginzburg, P.; Samusev, A.; Belov, P.; Valuckas, V.; Kuznetsov, A.I.; Luk'yanchuk, B.S.; Miroshnichenko, A.E.; et al. Probing Magnetic and Electric Optical Responses of Silicon Nanoparticles. Appl. Phys. Lett. 2015, 106, 171110. [CrossRef] 
52. Sinev, I.; Iorsh, I.; Bogdanov, A.; Permyakov, D.; Komissarenko, F.; Mukhin, I.; Samusev, A.; Valuckas, V.; Kuznetsov, A.I.; Luk'yanchuk, B.S.; et al. Polarization Control Over Electric and Magnetic Dipole Resonances of Dielectric Nanoparticles on Metallic Films. Laser Photonics Rev. 2016, 10, 799-806. [CrossRef]

53. Huang, Y.W.; Chen, W.T.; Wu, P.C.; Fedotov, V.; Savinov, V.; Ho, Y.Z.; Chau, Y.F.; Zheludev, N.I.; Tsai, D.P. Design of Plasmonic Toroidal Metamaterials at Optical Frequencies. Opt. Express 2012, 20, 1760-1768. [CrossRef] [PubMed]

54. Evlyukhin, A.B.; Fischer, T.; Reinhardt, C.; Chichkov, B.N. Optical Theorem and Multipole Scattering of Light by Arbitrarily Shaped Nanoparticles. Phys. Rev. B 2016, 94, 205434. [CrossRef]

55. Savinov, V.; Fedotov, V.A.; Zheludev, N.I. Toroidal Dipolar Excitation and Macroscopic Electromagnetic Properties of Metamaterials. Phys. Rev. B 2014, 89, 205112. [CrossRef]

56. Kaelberer, T.; Fedotov, V.A.; Papasimakis, N.; Tsai, D.P.; Zheludev, N.I. Toroidal Dipolar Response in a Metamaterial. Science 2010, 330, 1510-1512. [CrossRef]

(C) 2019 by the authors. Licensee MDPI, Basel, Switzerland. This article is an open access article distributed under the terms and conditions of the Creative Commons Attribution (CC BY) license (http:/ / creativecommons.org/licenses/by/4.0/). 